
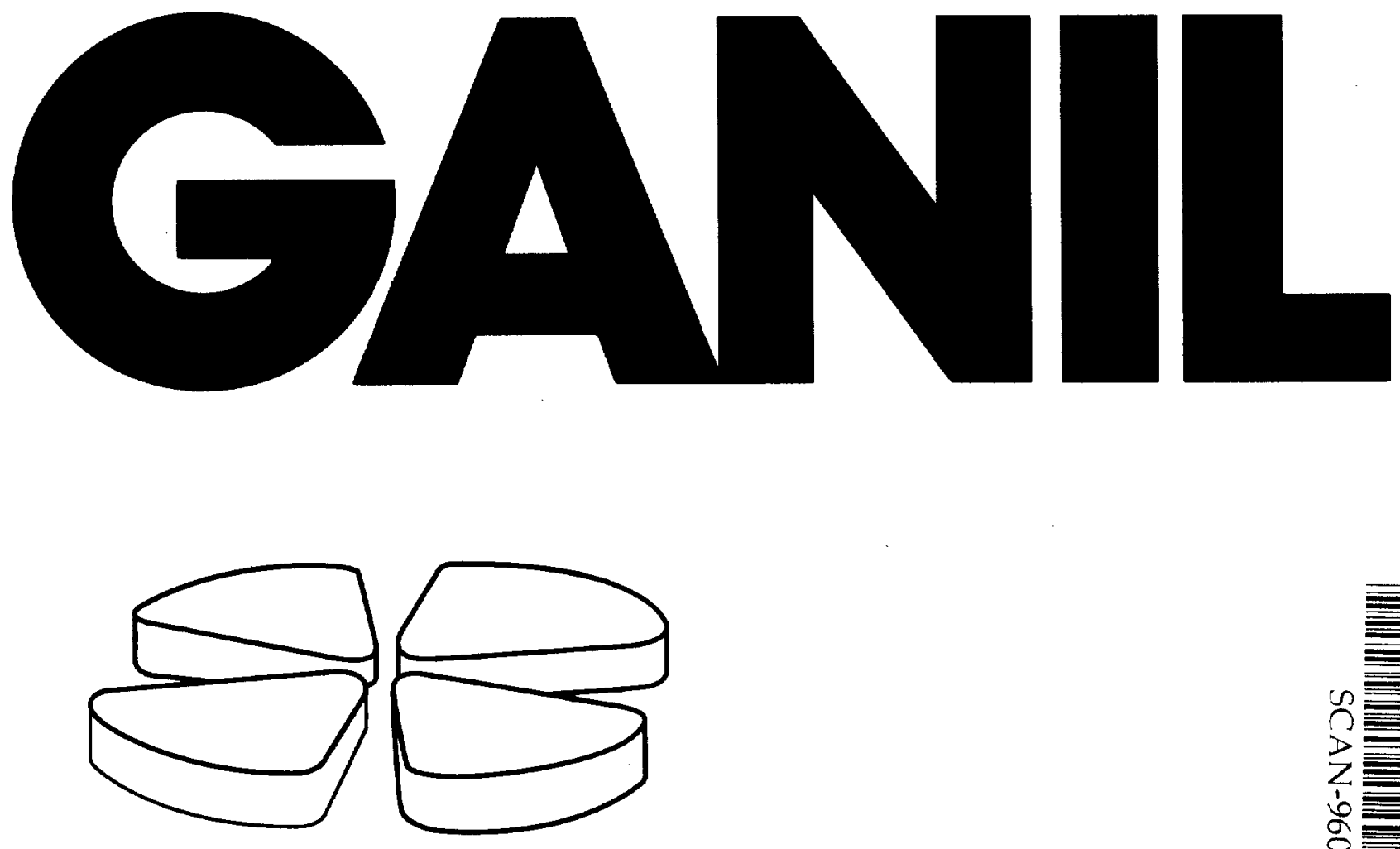

Beam Monitors Based On Residual Gas Ionization

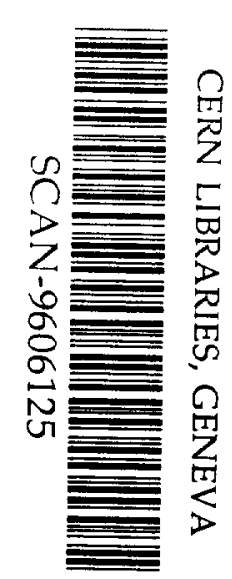

Jean-Luc P. Vignet, Rémy M. Anne, Yvon R. Georget, Robert E. Hue, Christian H. Tribouillard

Grand Accélérateur National d'Ions Lourds, BP 5027, 14021 Caen-cedex, France

Presented by Rémy M. Anne at the 7th Beam Instrumentation Workshop Argonne, Illinois (USA) May 6-9, 1996

GANIL P 9616 


\title{
Beam Monitors Based On Residual Gas Ionization
}

\author{
Jean-Luc P. Vignet, Rémy M. Anne, Yvon R. Georget, Robert E. Hue, \\ Christian $\mathrm{H}$. Tribouillard
}

Grand Accélérateur National d'Ions Lourds (GANIL), BP 5027, 14021 Caen-cedex, France

\begin{abstract}
At GANIL, we have developed two beam monitors based on ionization of the residual gas of the beam transport lines under the impact of the high energy heavy ion beams. One provides the beam profile i.e. its spatial distribution, the second one measures its time structure i.e. the width of the beam bunches delivered by the $10 \mathrm{Mhz}$ cyclotron. They are operated for beam intensities between a few nano-amperes and some micro-ampères and in a beam line vacuum better than $10^{-6}$ mbar. The charged ions or the electrons produced in the residual gas, drift by means of an electrostatic field onto a micro channel plate (M.C.P.), which amplifies the primary current. The M.C.P output signal is collected on a multistrip anode to get the beam profile, whereas the fast time structure of the beam is measured by means of a $50 \Omega$ anode. Calculations and results are presented as well as discussions about the different parameters of both devices.
\end{abstract}

\section{A NONINTERCEPTIVE BEAM PROFILE MONITOR}

\section{Introduction}

The profiles of the heavy ion beams at GANIL are usually measured by means of secondary emission multiwire monitors or by gas filled proportional chambers, according to the intensity of the beams. These beams range from Carbon to Uranium, and their intensities from a few hundred ions per second to some $\mu A e$. They provide the two horizontal and vertical profiles but, as they are interceptive, they deteriorate the beam properties or become themselves destroyed under high intensity beams. Thus, we have been developing and testing a noninterceptive profile monitor at GANIL, using the signal provided by the residual gas ionized under the passage of heavy ions (1).

\section{Monitor Principle}

All along the beam transfer lines, the pressure of which is around $10^{-6} \mathrm{mbar}$, ions are produced by ionization of the residual gas under the impact of the beam. If col- 


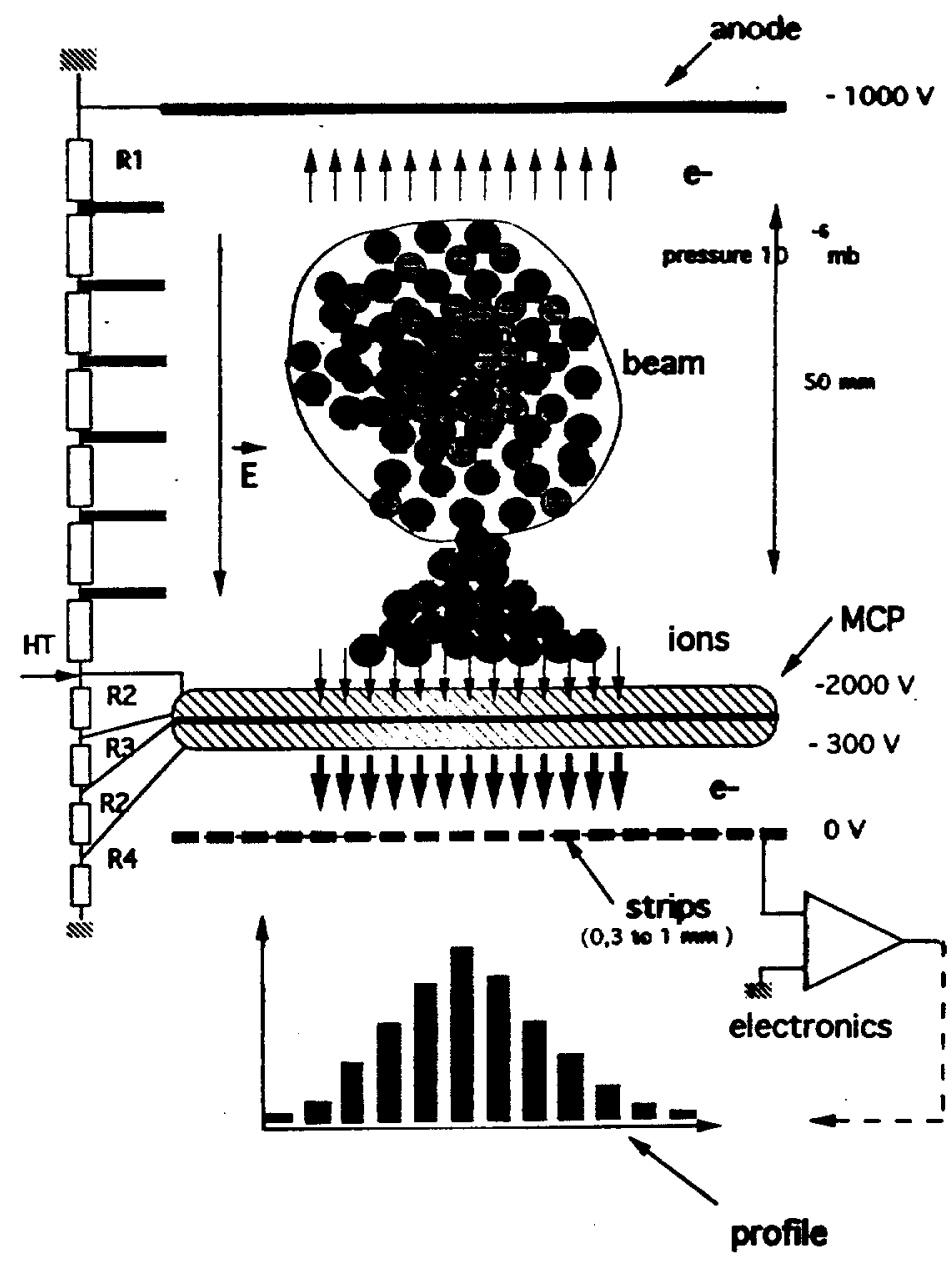

FIGURE 1 General layout of the monitor

lected, these ions, mainly hydrogen and water, provide information about the position and the size of the beam. Positive ions are guided by a transverse electrostatic field onto a microchannel plate (MCP) amplifier, the output of which is connected to a multistrip anode, thus providing signals proportional to the spatial beam density (Fig. 1). The number of ions produced in collisions between beam and residual gas depends on energy loss of the beam i.e. its nature and energy, but also on the pressure in the beam transfer lines. For example, in the case of a 200 nAe argon beam at $44 \mathrm{MeV} / \mathrm{u}$, circulating in a beam transfer line where the pressure is around $10^{-6} \mathrm{mbar}$, the positive ion current collected along $5 \mathrm{~mm}$ of the beam line is around 2 pAe.

\section{Mechanical Features}

The monitor is mainly composed of two parts, the drift space, and the amplifier stage. The $50 \mathrm{~mm}$ long electrostatic drift space for the residual gas ions, is obtained 
by means of a series of copper electrodes, the electrostatic potential of which is regularly distributed in order to have an homogeneous field in the central area. A stack of two micro-channel plates is used as amplifier, in front of which a mask limits a useful area, a few $\mathrm{mm}$ slit, perpendicular to the beam direction. The multistrip anode is made by gold deposit on a Teflon-glass support. The space $s$ between the centres of gravity of two contiguous strips can be as low as $0.2 \mathrm{~mm}$, which contributes to a good resolution of the beam size measurement.

\section{Electronics}

The charges collected on each anode strip at the output of the MCP are integrated by means of a capacity $C$ each one connected to an analog multiplexer which input impedance $R$ is high $\left(10^{9} \Omega\right.$ ). A $50 \mathrm{kHz}$ clock reads sequentially all the strips with a recurrence time $t_{i}$ which ranges between $10 \mathrm{~ms}$ and $5 \mathrm{~s}$, according to the beam intensity (2). This variable integration time, together with the adjustable gain of the $\mathrm{MCP}$, enables one to detect beams which intensity can vary in a large range Analog signals are converted in a microprocessor unit and sent to a computer, thus enabling one to do calculations on the profiles such as the centre of gravity and the width of the beam (Fig. 2).

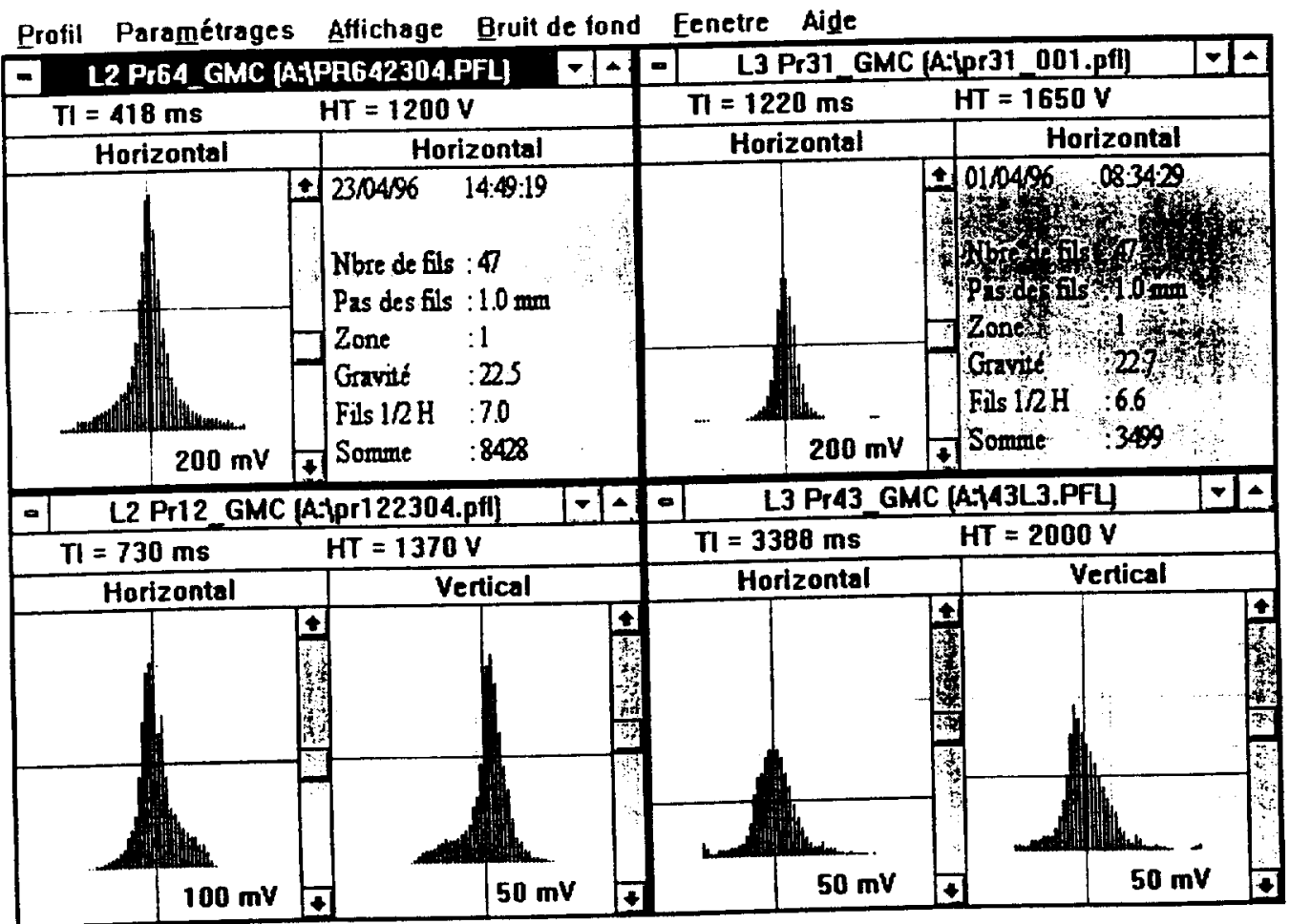

FIGURE 2 Beam profiles

FIGURE2a FIGURE 2b FIGURE $2 c$ FIGURE 2d
$\operatorname{Ar} 70 \mathrm{MeV} / \mathrm{u}, \mathrm{I}=2.6 \mu \mathrm{Ae}, \mathrm{s}=1 \mathrm{~mm}, \mathrm{Ti}=418 \mathrm{~ms}, \mathrm{HV}=1200 \mathrm{~V}$

Sn $75 \mathrm{MeV} / \mathrm{u}, \mathrm{I}=300 \mathrm{nAe}, \mathrm{s}=1 \mathrm{~mm}, \mathrm{Ti}=1220 \mathrm{~ms}, \mathrm{HV}=1650 \mathrm{~V}$

Sn $75 \mathrm{MeV} / \mathrm{u}, \mathrm{l}=300 \mathrm{nAe}, \mathrm{s}=0.5 \mathrm{~mm}, \mathrm{Ti}=730 \mathrm{~ms}, \mathrm{HV}=1370 \mathrm{~V}$

Te $40 \mathrm{MeV} / \mathrm{u}, \mathrm{I}=1 \mathrm{nAe}, \mathrm{s}=0.5 \mathrm{~mm}, \mathrm{Ti}=3388 \mathrm{~ms}, \mathrm{HV}=2000 \mathrm{~V}$ 


\section{Tests And Results}

We have performed a series of measurements using different beams of various intensities, pressure being better than $10^{-6}$ mbar. Some examples of profiles are displayed for the following beams, $\mathrm{Ar}, \mathrm{Sn}, \mathrm{Tc}$, and different parameters of the monitor and its associated electronics (Fig. 2a, b, c, d).

\section{Discussion}

Simulations have been carried out assuming that recoil ions are mainly produced in a $q=1$ charge state, that their kinetic energy is around $0.03 \mathrm{eV}$, and that they are isotropically emitted in a plane perpendicular to the beam direction (3). If $E$ is the electrostatic drift field, $m$ the mass of a recoil ion, $q$ its charge, $\theta$ and $E_{0}$ its angle and kinetic energy of emission, $t$ the time, $x$ and $y$ the axis respectively parallel and perpendicular to $E$, the motion equation of a recoil ion is the following one:

$$
\begin{aligned}
& x=\left(q E / 4 E_{0}\right) y^{2} / \sin ^{2} \theta+y / t g \theta+x_{0} \\
& y=\left(2 E_{0} / m\right)^{l / 2} t \sin \theta
\end{aligned}
$$

The trajectories of recoil ions emitted at different points of the volume of interaction of the beam with the residual gas, have been calculated, according to equations (1) with $\mathrm{E}_{0}=0.03 \mathrm{eV}, x_{0}=0$, and an angular spectrum $\theta=0$ to $360^{\circ} \mathrm{A}$ standard deviation has been estimated in these conditions $\sigma y=0.2 \mathrm{~mm}$ (Fig. 3).

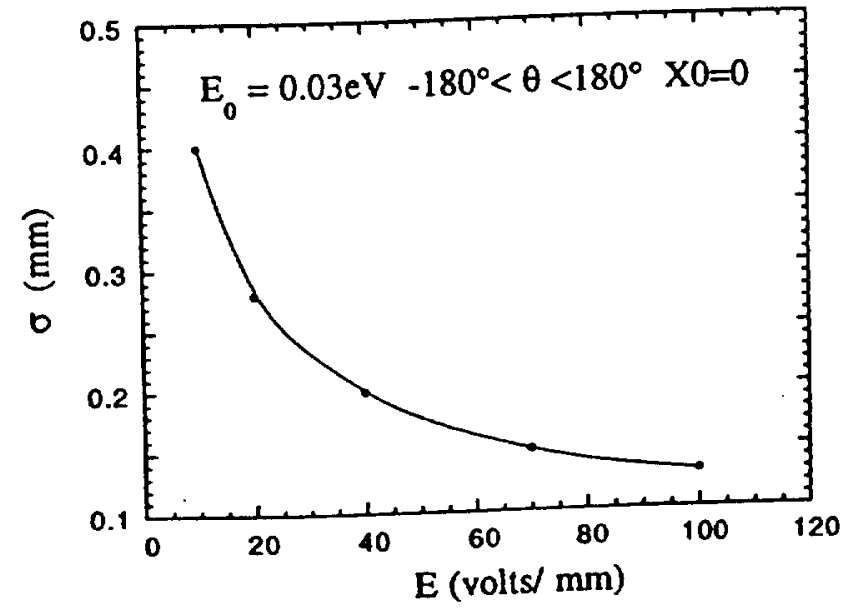

FIGURE 3 Theoretical standard deviation versus electrostatic drift field 


\section{BEAM TIME-STRUCTURE MONITOR}

\section{Introduction}

The GANIL cyclotrons deliver heavy ion bunched beams, the frequence of which is in-between 8 and $14 \mathrm{Mhz}$, and the bunch length less than 2 or 3 nanoseconds, depending on the energy and nature of the accelerated ions. The HF signal taken from the resonant cyclotron cavitics (Fig. 4a), is usually used for nuclei velocity measurements based on "time of flight" or "start-stop" technics, involved in reaction products identification processes. As a consequence, as short as possible beam bunches are needed. The aim of the monitor is to measure and check on line, the time distribution of the accelerated bunched beams.

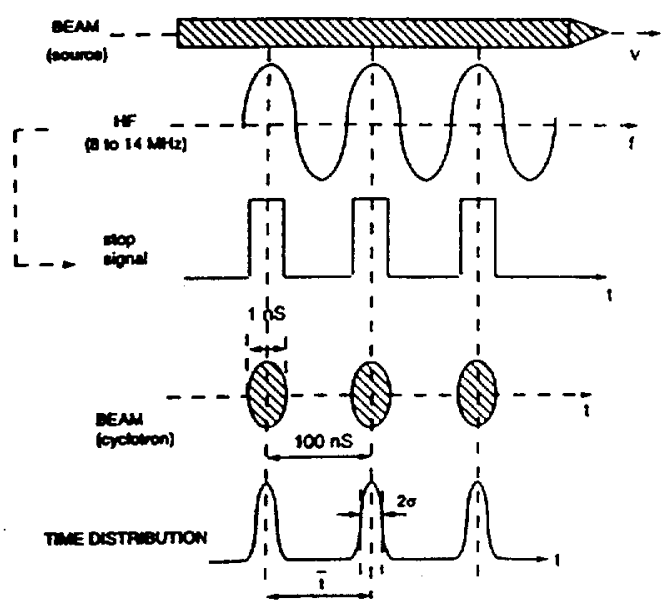

FIGURE $4 a$ timing of the GANIL beam

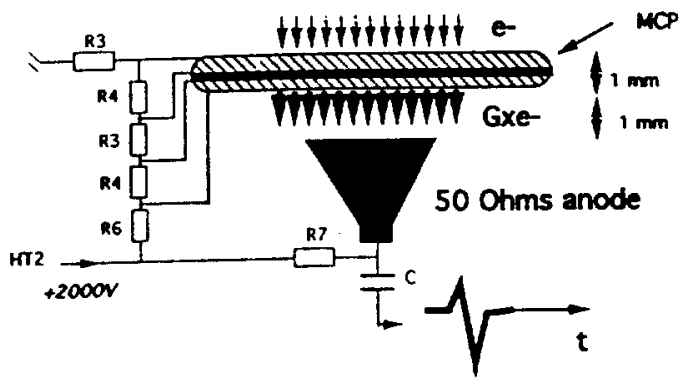

FIGURE $4 b$ output electronics

\section{Principle}

The signal is obtained from residual gas ionization, in the same conditions as previously described for the profile monitor, the localization strips being replaced by a $50 \Omega$ anode (Fig. 4 b).

According to the direction of the transverse electrostatic field $E$, electrons or ions produced by ionization of the residual gas under the impact of ion beams, are guided onto a microchannel plate amplifier which provides a signal, characteristic of the timing of the beam.

\section{Expected Resolution}

With the above mentioned parameters, the general motion equation of recoil ions or electrons produced by ionization of the residual gas, in the transverse electro- 
static drift field of the detector is :

$$
\mathrm{t}=\left(-\left(\mathrm{E}_{0}\right)^{1 / 2} \cdot \cos \theta+\left(\mathrm{E}_{0} \cos ^{2} \theta+\mathrm{qE}\left(\mathrm{d}-\mathrm{x}_{0}\right)\right)^{1 / 2}\right)(2 \mathrm{~m})^{1 / 2} / \mathrm{qE} c
$$

with $m$ and $\mathrm{E}_{0}$ in $\mathrm{eV}, q$ in charge units and $E$ in $\mathrm{V} / \mathrm{m}$. Ions or electrons can be collected, which of them provides the best resolution? According to vacuum gas analysis most part of residual gas is hydrogen and for a smaller part, water. So, we will compare for example, the motion of an atom of ionized hydrogen with the one of an electron, and calculate for the in both of them, the time dispersion motion $\Delta t$ according to different emission parameters, specially their $x_{0}$ coordinate. We will take for example a beam, the size of which is $W=4 \mathrm{~mm}$, the distance to the microchannel plate is $d=30 \mathrm{~mm}$, a mean kinetic energy of $0.03 \mathrm{eV}$ (9) and 2.0 $\mathrm{eV}$ for respectively the emitted hydrogen ion and electron, and suppose in all cases: $\theta=0^{\circ}, E=40 \mathrm{~V} / \mathrm{mm}, \mathrm{x}_{0}= \pm \mathrm{W} / 2= \pm 2 \mathrm{~mm}$. The derivative of $\mathrm{t}$, versus $\mathrm{x}=\left(\mathrm{d}-\mathrm{x}_{0}\right)$ gives:

$$
\mathrm{dt} / \mathrm{d} \mathrm{x}_{0}=(1 / 2 \mathrm{c})\left(2 \mathrm{~m} /\left(\mathrm{E} 0+\mathrm{qE}\left(\mathrm{d}-\mathrm{x}_{0}\right)\right) 1 / 2\right.
$$

which results in $\Delta \mathrm{t}$ (hydrogen $)=3.1210^{-9} \mathrm{sec}$ and $\Delta \mathrm{t}$ (electron) $=7410^{-12} \mathrm{sec}$. This succinct calculation indicates that it is much better to take the time signal from electrons than from hydrogen ions, specially if in addition we notice that the ions from the residual gas are not only hydrogen but heavier atoms like water, carbone oxyde, nitrogen, argon and so on.

\section{Resolution as a function of :}

\section{Beam-size}

Equation (4) with the above parameters values, shows that for electrons, $\Delta t=18$ $10^{-12} \mathrm{sec}$ for $1 \mathrm{~mm}$ beam size. The variation of $\Delta t$ for a 0 to $6 \mathrm{~mm}$ beam size is represented (Fig.5a).

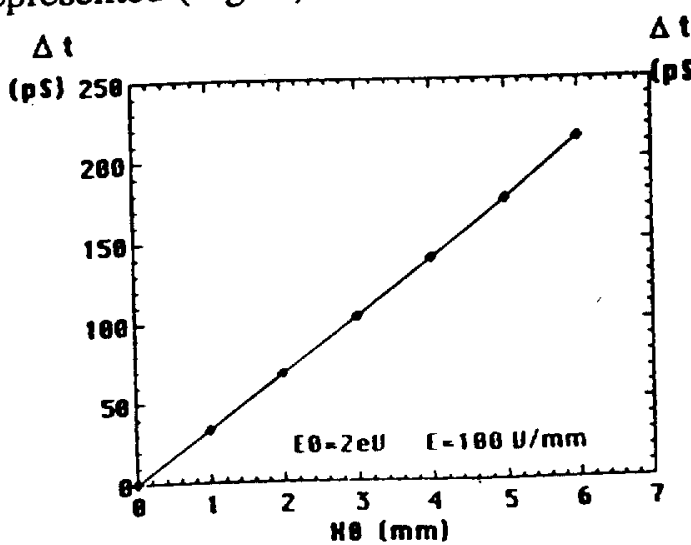

Figure 5 a $\Delta t$ versus beam size
$\Delta t$

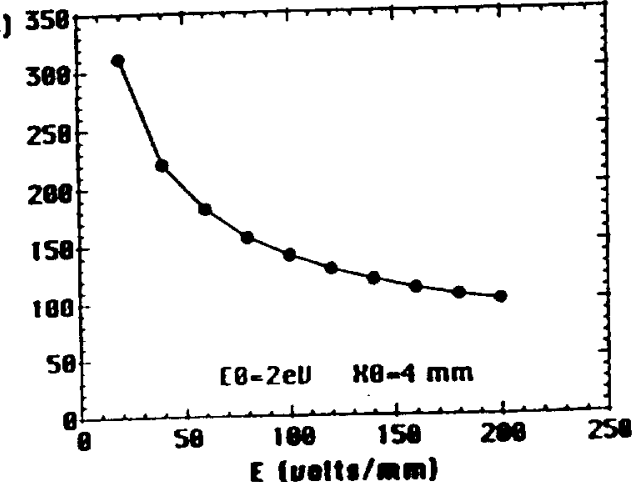

FIGURE 5b $\Delta t$ versus electrostatic field 


\section{Beam velocity}

In order to reduce the time gitter, we delimil the active area of the monitor by a 3 to $5 \mathrm{~mm}$ width slit $l$, perpendicular to the beam direction. For example, with $\beta=$ $0.2 \mathrm{~d} v \mathrm{dl}=16.710^{-12} \mathrm{sec} / \mathrm{mm}$.

\section{Electrostatic field}

$\mathrm{dt} / \mathrm{dE}=(2 \mathrm{mE} 0)^{1 / 2} \cdot \mathrm{E}^{-2} / \mathrm{qc}-\left((2 \mathrm{~m})^{1 / 2} / 2 \mathrm{qc}\right)\left(2 \mathrm{E}_{0} \mathrm{E}^{-3}+\mathrm{q}\left(\mathrm{d}-\mathrm{x}_{0}\right) \mathrm{E}^{-2}\right)$

$\left(\mathrm{E}_{0} \mathrm{E}^{-2}+\mathrm{q}\left(\mathrm{d}-\mathrm{x}_{0}\right) \mathrm{E}^{-1}\right)^{-1 / 2}$

with $d=30 \mathrm{~mm}, E=10^{5} \mathrm{~V} / \mathrm{m}, E_{0}=2 \mathrm{eV}(\mathrm{dt} / \mathrm{dE})=-0.510^{-12} \mathrm{~V} / \mathrm{m}$

which shows that a as high as possible ficld $E$ is required as it can be seen on figure 5b. For a gaussian beam, $E=100 \mathrm{~V} / \mathrm{mm}, x_{0}=4 \mathrm{~mm}$, the standard deviation is around $2510^{-12} \mathrm{sec}$.

\section{Distance $d$ between the beam axis and the microchannel plate}

The distance $d$ is in fact limited by a compromise between the maximum high voltage $\mathrm{V}$ that can be applied to the device, the minimum distance $d$ between the beam axis and the MCP, and the needed high value of $E=\mathrm{V} / \mathrm{d}$

We have chosen $d=30 \mathrm{~mm}$, even though in the case of a larger beam size, $d$ should be higher in order to increase the resolution.

\section{Electronics}

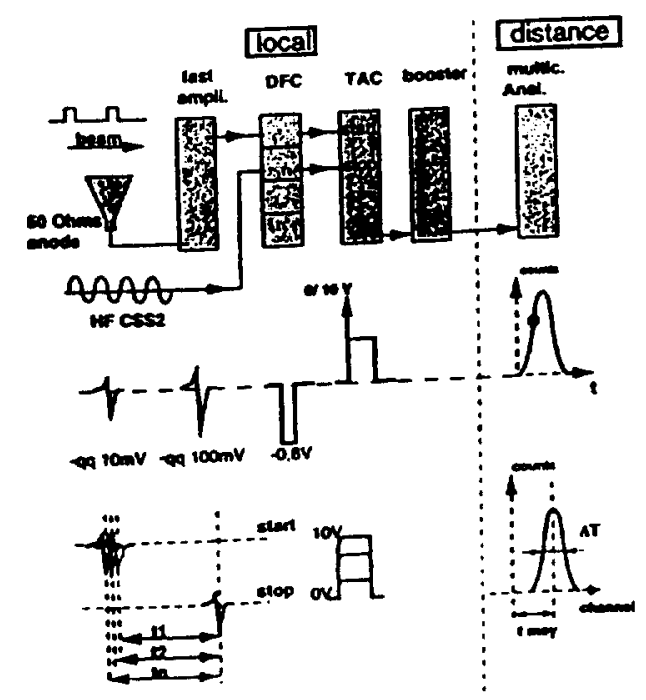

FIGURE 6 signal processing synoptic 
Each electron produced in the vacuum is sent onto the MCP stack, giving rise to a fast signal which charge is equal to $G \mathrm{e}^{-}$. This signal, collected on a $50 \Omega$ anode (Fig. 6), is first amplified, sent in a constant fraction discriminator and finally in a time amplitude converter (TAC) as start signal. the stop being taken as the cyclotron HF signal. The TAC output signal, sent in a multichannel analyser, provides the time distribution of the cyclotron beam bunches.

\section{Results}

On line measurements have been performed during physics experiments in order to check the stability of the beam (Fig. 7a, b, c), or during cyclotron beam tuning (Fig. 7d).

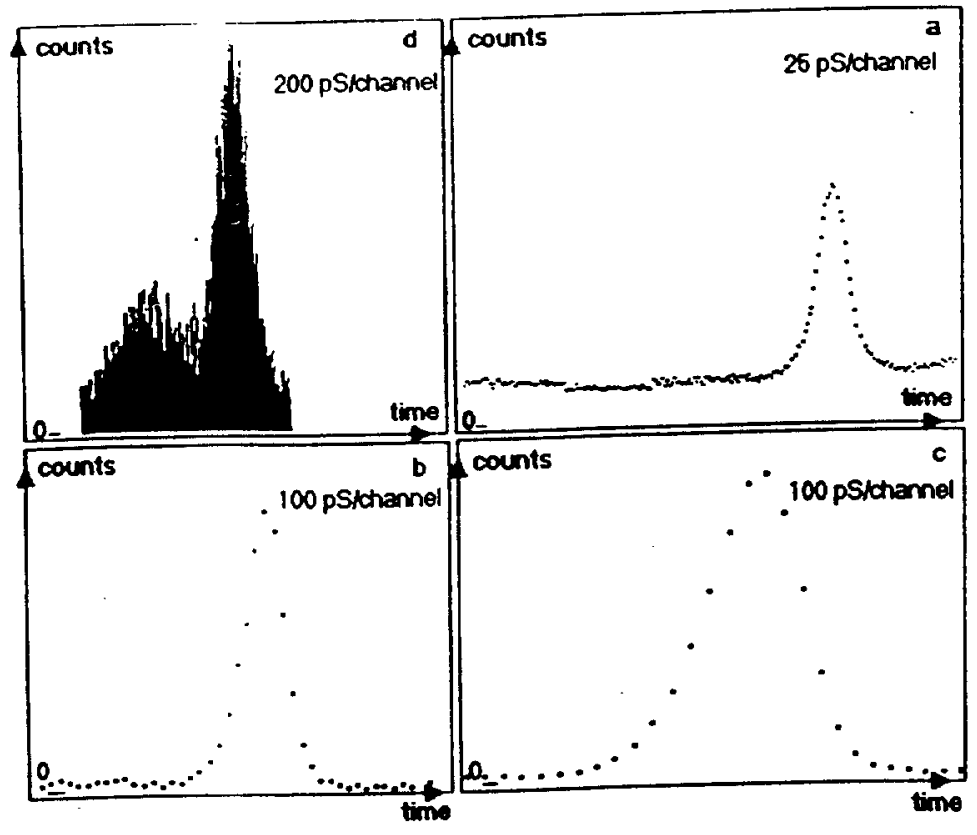

FIGURE 7a Sn $63 \mathrm{MeV} / \mathrm{u}, \mathrm{l}=350 \mathrm{nAe}, \mathrm{Ti}=300 \mathrm{sec}, \Delta \mathrm{t}(\mathrm{fwmh})=1.5 \mathrm{nS}$

FIGURE $7 \mathrm{~b} O 63 \mathrm{MeV} / \mathrm{u}, \mathrm{I}=1.7 \mu \mathrm{Ae}, \mathrm{t} i=560 \mathrm{sec}, \Delta \mathrm{t}=550 \mathrm{ps}$

FIGURE 7c O $63 \mathrm{MeV} / \mathrm{u}, \mathrm{I}=200 \mathrm{nAe}, \mathrm{t} i=7600 \mathrm{sec}, \Delta \mathrm{t}=600 \mathrm{ps}$

FIGURE 7d Ar $30 \mathrm{MeV} / \mathrm{u}, \mathrm{I}=90 \mathrm{nAe}, \Delta \mathrm{t}=900 \mathrm{ps}$

\section{REFERENCES}

1. Anne R.M., Georget Y.R., Hue, Tribouillard C.H, Vignet J.L.P. Rapport GANIL, R 87-10

2. Anne R.M., Georget Y.R., Hue, Tribouillard C.H, Vignet J.L.P. A noninterceptive heavy ion beam profile monitor based on residual gas ionization, NIM A329, 21-28, North-Holland (1993).

3. Grandin J.P., Hennecard D., Husson X., Leclerc D., Lesteven-Vaisse, and Lisfi D. EUROPHYS.LETT. 6(8) 683 (1988) 